\title{
Quantum Theory Approach for Neutron Single and Double-Slit Diffraction
}

\author{
Xiang-Yao $\mathrm{Wu}^{a}{ }^{*}$, Bai-Jun Zhang ${ }^{a}$, Zhong $\mathrm{Hua}^{a}$ \\ Xiao-Jing Liu ${ }^{a}$, Yi-Heng $\mathrm{Wu}^{a}$, Hou-Li Tang ${ }^{a}$ and JIng-Wu Li ${ }^{b}$ \\ a.Institute of Physics, Jilin Normal University, Siping 136000, China \\ b.Institute of Physics, Xuzhou Normal University, Xuzhou 221000, China
}

\begin{abstract}
We provide a quantum approach description of neutron single and double-slit diffraction, with specific attention to the cold neutron diffraction $(\lambda \approx 20 \AA)$ carried out by Zeilinger et al. in 1988 . We find the theoretical results are good agreement with experimental data.

PACS: 03.75.Dg, 03.65.Ta, 03.65.Yz

Keywords: Neutron diffraction; Quantum theory; Decoherence effect
\end{abstract}

\section{Introduction}

The matter-wave diffraction has become a large field of interest over the last years, and it is extended to electron, neutron, atom, more massive, complex objects, like large molecules $I_{2}, C_{60}$ and $C_{70}$, which were found in experiments [1-5]. At present, There are classical and quantum methods to study interference and diffraction [6-12]. As is well known, the classical optics with its standard wave-theoretical methods and approximations, in particular those of Huygens and Kirchhoff, has been successfully applied to classical optics, and has yielded good agreement with many experiments. This simple wave-optical approach also gives a description of matter wave diffraction. However, matter-wave interference and diffraction are quantum phenomena, and its full description needs quantum mechanical approach. Recently, there are some quantum theory approach to study electron and neutron diffraction, and obtain some important and new results [13-17]. In viewpoint of quantum mechanics, the neutron has the wave nature, which is described by wave function $\psi(\vec{r}, t)$, and the wave function $\psi(\vec{r}, t)$ has statistical meaning, i.e., $|\psi(\vec{r}, t)|^{2}$ can be explained as particle's probability density. For the single and double-slit diffraction, if we can calculate the neutron wave function $\psi(\vec{r}, t)$ distributing on display screen, then we can obtain the diffraction intensity, since the diffraction intensity is directly proportional to $|\psi(\vec{r}, t)|^{2}$. In the single and double-slit diffraction, the neutron wave functions can be divided into three parts. The first is the incident area, and the neutron wave function is a plane wave. The second is the slit area, where the neutron wave function can be calculated by the Schrödinger wave equation. The third is the diffraction area, where the neutron wave function can be obtained by Kirchhoff's law. Otherwise, we consider the decoherence effect in the double slit diffraction. We know decoherence is the irreversible emergence of classical properties when an isolated system interacts with an environment [18]. The environment can be constituted by many randomly distributed particles interacting with the system by means of scattering processes. When these events occur in a large number, the off-diagonal elements of the system reduced density matrix undergo an exponential damping [19], this making the system to quickly lose its coherence, i.e., the decoherence is the dynamic suppression of the interference terms owing to the interaction between system and environment. In this paper, we study the neutron single and double-slit diffraction with the quantum approach, and analyze the influence of the decoherence machanism to the double slit diffraction. We compare our calculation results to the cold neutron $(\lambda \approx 20 \AA)$ diffraction experiment carried out by Zeilinger et al. in 1988 [20]. We find the decoherence machanism has improved the calculation result of the double slit diffraction, and the theory results are agreement with the experiment data.

* E-mail: wuxy2066@163.com 
FIG. 1: Double-slit geometry with $a_{1}$ the first slit width, $a_{2}$ the second slit width, $b$ the slit length and $d$ the distance between the two slits.

\section{Quantum approach of neutron diffraction}

In an infinite plane, we consider a double-slit, its width $a_{1}$ and $a_{2}$, length $b$, thickness $c$ and the slit-to-slit distance $d$ are shown in FIG. 1 . The $x$ axis is along the slit length and the $y$ axis is along the slit width. We calculate the neutron wave function in the first single slit (left) with the Schrödinger equation, and the neutron wave function of the second single-slit (right) can be obtained easily. At time $t$, we suppose that the incident plane wave travels along the $z$ axis. It is

$$
\psi_{0}(z, t)=A e^{\frac{i}{\hbar}(p z-E t)}
$$

where $A$ is plane wave amplitude.

The potential in the first single slit is

$$
V(x, y, z)=\left\{\begin{array}{lc}
0 & 0 \leq x \leq b, 0 \leq y \leq a_{1}, 0 \leq z \leq c, \\
\infty & \text { otherwise }
\end{array}\right.
$$

where $c$ is the thickness of the single slit. The time-dependent and time-independent Schrödinger equations are

$$
\begin{gathered}
i \hbar \frac{\partial}{\partial t} \psi(\vec{r}, t)=-\frac{\hbar^{2}}{2 M}\left(\frac{\partial^{2}}{\partial x^{2}}+\frac{\partial^{2}}{\partial y^{2}}+\frac{\partial^{2}}{\partial z^{2}}\right) \psi(\vec{r}, t), \\
\frac{\partial^{2} \psi(\vec{r})}{\partial x^{2}}+\frac{\partial^{2} \psi(\vec{r})}{\partial y^{2}}+\frac{\partial^{2} \psi(\vec{r})}{\partial z^{2}}+\frac{2 M E}{\hbar^{2}} \psi(\vec{r})=0,
\end{gathered}
$$

where $M(E)$ is the mass(energy) of the neutron. The relation between $\psi(\vec{r}, t)$ and $\psi(\vec{r})$ is

$$
\psi(x, y, z, t)=\psi(x, y, z) e^{-\frac{i}{\hbar} E t}
$$

In Eq. (4), the wave function $\psi(x, y, z)$ satisfies the boundary conditions

$$
\begin{gathered}
\psi(0, y, z)=\psi(b, y, z)=0 \\
\psi(x, 0, z)=\psi\left(x, a_{1}, z\right)=0 .
\end{gathered}
$$

The Eq. (4) can be solved by the method of separation of variable. By writing

$$
\psi(x, y, z)=X(x) Y(y) Z(z)
$$


The general solution of Eq. (3) is

$$
\begin{aligned}
\psi_{1}(x, y, z, t) & =\sum_{m n} \psi_{m n}(x, y, z, t) \\
& =\sum_{m n} D_{m n} \sin \frac{n \pi x}{b} \sin \frac{m \pi y}{a_{1}} e^{i \sqrt{\frac{2 M E}{\hbar^{2}}-\frac{n^{2} \pi^{2}}{b^{2}}-\frac{m^{2} \pi^{2}}{a_{1}^{2}}} z} e^{-\frac{i}{\hbar} E t}
\end{aligned}
$$

Eq. (9) is the neutron wave function in the first single slit. Since the wave functions are continuous at $z=0$, we have

$$
\left.\psi_{0}(x, y, z, t)\right|_{z=0}=\left.\psi_{1}(x, y, z, t)\right|_{z=0}
$$

From Eqs. (2), (6) and (9), we can obtain the Fourier coefficient $D_{m n}$ by Fourier transform

$$
\begin{aligned}
D_{m n} & =\frac{4}{a_{1} b} \int_{0}^{a_{1}} \int_{0}^{b} A \sin \frac{n \pi \xi}{b} \sin \frac{m \pi \eta}{a_{1}} d \xi d \eta \\
& =\left\{\begin{array}{lc}
\frac{16 A}{m n \pi^{2}} & \text { m, n, odd, } \\
0 & \text { otherwise, }
\end{array}\right.
\end{aligned}
$$

substituting Eq. (11) into Eq. (9), we can obtain the neutron wave function in the first single slit.

$$
\begin{aligned}
\psi_{1}(x, y, z, t)= & \sum_{m, n=0}^{\infty} \frac{16 A}{(2 m+1)(2 n+1) \pi^{2}} \sin \frac{(2 n+1) \pi x}{b} \sin \frac{(2 m+1) \pi y}{a_{1}} \\
& \cdot e^{i \sqrt{\frac{2 M E}{\hbar^{2}}-\frac{(2 n+1)^{2} \pi^{2}}{b^{2}}-\frac{(2 m+1)^{2} \pi^{2}}{a_{1}^{2}}} z} e^{-\frac{i}{\hbar} E t} .
\end{aligned}
$$

The neutron wave function in the second single slit can be obtained by making the coordinate translations $x^{\prime}=x, y^{\prime}=y-a_{1}-d, z^{\prime}=z$, and we can obtain the neutron wave function $\psi_{2}(x, y, z, t)$ in the second slit

$$
\begin{aligned}
\psi_{2}(x, y, z, t)= & \sum_{m, n=0}^{\infty} \frac{16 A}{(2 m+1)(2 n+1) \pi^{2}} \sin \frac{(2 n+1) \pi x}{b} \sin \frac{(2 m+1) \pi\left(y-a_{1}-d\right)}{a_{2}} \\
& \cdot e^{i \sqrt{\frac{2 M E}{\hbar^{2}}-\frac{(2 n+1)^{2} \pi^{2}}{b^{2}}-\frac{(2 m+1)^{2} \pi^{2}}{a_{2}^{2}}} z} e^{-\frac{i}{\hbar} E t} .
\end{aligned}
$$

\section{The wave function of neutron diffraction}

With Kirchhoff's law, we can calculate the neutron wave function in the diffraction area. It can be calculated by the formula [21]

$$
\psi_{\text {out }}(\boldsymbol{r}, t)=-\frac{1}{4 \pi} \int_{s} \frac{e^{i k r}}{r} \boldsymbol{n} \cdot\left[\nabla^{\prime} \psi_{\text {in }}+\left(i k-\frac{1}{r}\right) \frac{\boldsymbol{r}}{r} \psi_{i n}\right] d s,
$$

where $\psi_{\text {out }}(\boldsymbol{r}, t)$ is the diffraction wave function on display screen, $\psi_{\text {in }}(\boldsymbol{r}, t)$ is the wave function of slit surface $(z=c)$ and $s$ is the area of the aperture or slit.

For the double-slit diffraction, Eq. (14) becomes

$$
\begin{aligned}
\psi_{\text {out }}(\boldsymbol{r}, t)= & -\frac{1}{4 \pi} \int_{s_{1}} \frac{e^{i k r}}{r} n \cdot\left[\nabla^{\prime} \psi_{1}+\left(i k-\frac{1}{r}\right) \frac{\boldsymbol{r}}{r} \psi_{1}\right] d s \\
& -\frac{1}{4 \pi} \int_{s_{2}} \frac{e^{i k r}}{r} n \cdot\left[\nabla^{\prime} \psi_{2}+\left(i k-\frac{1}{r}\right) \frac{\boldsymbol{r}}{r} \psi_{2}\right] d s .
\end{aligned}
$$

In Eq. (15), the first and second terms are corresponding to the diffraction wave functions of the first slit and second slit. 


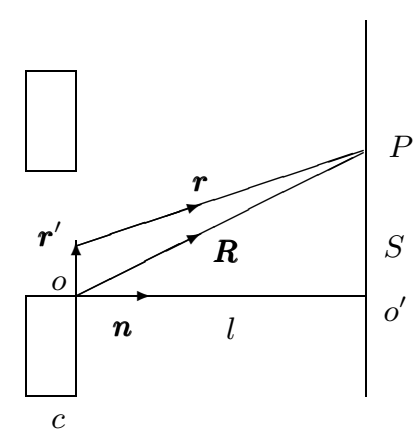

FIG. 2: Diffraction area of the single slit

In the following, we firstly calculate the diffraction wave function of the first slit, it is

$$
\psi_{\text {out }_{1}}(\boldsymbol{r}, t)=-\frac{1}{4 \pi} \int_{s_{1}} \frac{e^{i k r}}{r} \boldsymbol{n} \cdot\left[\nabla^{\prime} \psi_{1}+\left(i k-\frac{1}{r}\right) \frac{\boldsymbol{r}}{r} \psi_{1}\right] d s .
$$

The diffraction area is shown in FIG. 2, where $k=\sqrt{\frac{2 M E}{\hbar^{2}}}, s_{1}$ is the area of the first single-slit, $\boldsymbol{r}^{\prime}$ is the position of a point on the surface $(\mathrm{z}=\mathrm{c}), P$ is an arbitrary point in the diffraction area, and $\boldsymbol{n}$ is a unit vector, which is normal to the surface of the slit.

From FIG. 2, we have

$$
\begin{aligned}
r & =R-\frac{\boldsymbol{R}}{R} \cdot \boldsymbol{r}^{\prime} \approx R-\frac{\boldsymbol{r}}{r} \cdot \boldsymbol{r}^{\prime} \\
& =R-\frac{\boldsymbol{k}_{2}}{k} \cdot \boldsymbol{r}^{\prime}
\end{aligned}
$$

and then,

$$
\begin{aligned}
\frac{e^{i k r}}{r} & =\frac{e^{i k\left(R-\frac{\boldsymbol{r}}{r} \cdot \boldsymbol{r}^{\prime}\right)}}{R-\frac{\boldsymbol{r}}{r} \cdot \boldsymbol{r}^{\prime}}=\frac{e^{i k R} e^{-i \boldsymbol{k}_{2} \cdot \boldsymbol{r}^{\prime}}}{R-\frac{\boldsymbol{r}}{r} \cdot \boldsymbol{r}^{\prime}} \\
& \approx \frac{e^{i k R} e^{-i \boldsymbol{k}_{2} \cdot \boldsymbol{r}^{\prime}}}{R} \quad\left(\left|\boldsymbol{r}^{\prime}\right| \ll R\right),
\end{aligned}
$$

with $\boldsymbol{k}_{2}=k \frac{\boldsymbol{r}}{r}$. Substituting Eq. (17) and (18) into Eq. (16), one can obtain

$$
\begin{aligned}
\psi_{\text {out }_{1}}(\boldsymbol{r}, t)= & -\frac{e^{i k R}}{4 \pi R} e^{-\frac{i}{\hbar} E t} \int_{s_{0}} e^{-i \boldsymbol{k}_{2} \cdot \boldsymbol{r}^{\prime}} \sum_{m=0}^{\infty} \sum_{n=0}^{\infty} \frac{16 A}{(2 m+1)(2 n+1) \pi^{2}} \\
& \cdot e^{i \sqrt{\frac{2 M E}{\hbar^{2}}-\left(\frac{(2 n+1) \pi}{b}\right)^{2}-\left(\frac{(2 m+1) \pi}{a_{1}}\right)^{2}} \cdot c} \sin \frac{(2 n+1) \pi}{b} x^{\prime} \sin \frac{(2 m+1) \pi}{a_{1}} y^{\prime} \\
& \cdot\left[i \sqrt{\frac{2 M E}{\hbar^{2}}-\left(\frac{(2 n+1) \pi}{b}\right)^{2}-\left(\frac{(2 m+1) \pi}{a_{1}}\right)^{2}}+i \boldsymbol{n} \cdot \boldsymbol{k}_{2}-\frac{\boldsymbol{n} \cdot \boldsymbol{R}}{R^{2}}\right] d x^{\prime} d y^{\prime} .
\end{aligned}
$$

Assume that the angle between $\boldsymbol{k}_{2}$ and $x$ axis $\left(y\right.$ axis) is $\frac{\pi}{2}-\alpha\left(\frac{\pi}{2}-\beta\right)$, and $\alpha(\beta)$ is the angle between $\boldsymbol{k}_{2}$ and the surface of $y z(x z)$, then we have

$$
\begin{gathered}
k_{2 x}=k \sin \alpha, \quad k_{2 y}=k \sin \beta, \\
\boldsymbol{n} \cdot \boldsymbol{k}_{2}=k \cos \theta,
\end{gathered}
$$

where $\theta$ is the angle between $\boldsymbol{k}_{2}$ and $z$ axis, and the angles $\theta, \alpha, \beta$ satisfy the equation

$$
\cos ^{2} \theta+\cos ^{2}\left(\frac{\pi}{2}-\alpha\right)+\cos ^{2}\left(\frac{\pi}{2}-\beta\right)=1
$$

From FIG. 2, we have

$$
\sin \beta=\frac{s}{R},
$$


with $R=\sqrt{l^{2}+s^{2}}$. Substituting Eqs. (20)-(23) into Eq. (19) yields

$$
\begin{aligned}
\psi_{\text {out }_{1}}(x, y, z, t)= & -\frac{e^{i k R}}{4 \pi R} e^{-\frac{i}{\hbar} E t} \sum_{m=0}^{\infty} \sum_{n=0}^{\infty} \frac{16 A}{(2 m+1)(2 n+1) \pi^{2}} e^{i \sqrt{\frac{2 M E}{\hbar^{2}}-\left(\frac{(2 n+1) \pi}{b}\right)^{2}-\left(\frac{(2 m+1) \pi}{a_{1}}\right)^{2} \cdot c}} \\
& \cdot\left[i \sqrt{\frac{2 M E}{\hbar^{2}}-\left(\frac{(2 n+1) \pi}{b}\right)^{2}-\left(\frac{(2 m+1) \pi}{a_{1}}\right)^{2}}+\left(i k-\frac{1}{R}\right) \sqrt{\cos ^{2} \alpha-\left(\frac{s}{R}\right)^{2}}\right] \\
& \cdot \int_{0}^{b} e^{-i k \sin \alpha \cdot x^{\prime}} \sin \frac{(2 n+1) \pi}{b} x^{\prime} d x^{\prime} \int_{0}^{a_{1}} e^{-i k \sin \beta \cdot y^{\prime}} \sin \frac{(2 m+1) \pi}{a_{1}} y^{\prime} d y^{\prime} .
\end{aligned}
$$

Equation (24) is the diffraction wave function of the first slit. The neutron diffraction wave-function for the second slit can be obtained by making the coordinate translations $x^{\prime}=x, y^{\prime}=y-(a+d), z^{\prime}=z$, it is

$$
\begin{aligned}
\psi_{\text {out }_{2}}(x, y, z, t)= & -\frac{e^{i k R}}{4 \pi R} e^{-\frac{i}{\hbar} E t} \sum_{m=0}^{\infty} \sum_{n=0}^{\infty} \frac{16 A}{(2 m+1)(2 n+1) \pi^{2}} e^{i \sqrt{\frac{2 M E}{\hbar^{2}}-\left(\frac{(2 n+1) \pi}{b}\right)^{2}-\left(\frac{(2 m+1) \pi}{a_{2}}\right)^{2} \cdot c}} \\
& {\left[i \sqrt{\frac{2 M E}{\hbar^{2}}-\left(\frac{(2 n+1) \pi}{b}\right)^{2}-\left(\frac{(2 m+1) \pi}{a_{2}}\right)^{2}}+\left(i k-\frac{1}{R}\right) \sqrt{\left.\cos ^{2} \alpha-\left(\frac{s}{R}\right)^{2}\right]}\right.} \\
& \int_{0}^{b} e^{-i k \sin \alpha \cdot x^{\prime}} \sin \frac{(2 n+1) \pi}{b} x^{\prime} d x^{\prime} \\
& \int_{a_{1}+d}^{a_{1}+a_{2}+d} e^{-i k \sin \beta \cdot y^{\prime}} \sin \frac{(2 m+1) \pi}{a_{2}}\left(y^{\prime}-\left(a_{1}+d\right)\right) d y^{\prime},
\end{aligned}
$$

where $d$ is the two slit distance. The total diffraction wave function for the double-slit is

$$
\psi_{\text {out }}(x, y, z, t)=c_{1} \psi_{\text {out }_{1}}(x, y, z, t)+c_{2} \psi_{\text {out }_{2}}(x, y, z, t)
$$

where $c_{1}$ and $c_{2}$ are superposition coefficients, and $\left|c_{1}\right|^{2}+\left|c_{2}\right|^{2}=1$. For the single-slit diffraction, we can obtain the relative diffraction intensity $I$ on the display screen,

$$
I \propto\left|\psi_{\text {out } 1}(x, y, z, t)\right|^{2}
$$

For the double-slit diffraction, we can obtain the relative diffraction intensity $I$ on the display screen,

$$
\begin{aligned}
I & \propto\left|\psi_{\text {out }}(x, y, z, t)\right|^{2} \\
& =c_{1}^{2}\left|\psi_{\text {out } 1}(x, y, z, t)\right|^{2}+c_{2}^{2}\left|\psi_{\text {out } 2}(x, y, z, t)\right|^{2}+2 c_{1} c_{2} \operatorname{Re}\left[\psi_{\text {out } 1}^{*}(x, y, z, t) \psi_{\text {out } 2}(x, y, z, t)\right] .
\end{aligned}
$$

\section{Decoherence effect in double-slit diffraction}

Decoherence is introduced here using a simple phenomenological theoretical model that assumes an exponential damping of the interferences $[7,18,19]$, i.e., the decoherence is the dynamic suppression of the interference terms owing to the interaction between system and environment. The Eq. (26) describes the coherence state coherence superposition, without considering the interaction of system with external environment. When we consider the effect of external environment, the total wave function of system and environment for the double-slit factorizes as [7]

$$
\psi_{\text {out }}(x, y, z, t)=c_{1} \psi_{\text {out }_{1}} \otimes\left|E_{1}>_{t}+c_{2} \psi_{\text {out }_{2}} \otimes\right| E_{2}>_{t}
$$

where $\mid E_{1}>_{t}$ and $\mid E_{2}>_{t}$ describe the state of the environment. The diffraction intensity on the screen is now given by[7]:

$$
I=\left(1+\left|\alpha_{t}\right|^{2}\right)\left(c_{1}^{2}\left|\psi_{\text {out } 1}(x, y, z, t)\right|^{2}+c_{2}^{2}\left|\psi_{\text {out } 2}(x, y, z, t)\right|^{2}+2 c_{1} c_{2} \Lambda_{t} \operatorname{Re}\left[\psi_{\text {out } 1}^{*}(x, y, z, t) \psi_{\text {out } 2}(x, y, z, t)\right]\right) .(30)
$$


where $\alpha_{t}={ }_{t}<E_{2} \mid E_{1}>_{t}$, and $\Lambda_{t}=\frac{2\left|\alpha_{t}\right|}{1+\left|\alpha_{t}\right|^{2}}$. Thus, $\Lambda_{t}$ is defined as the quantum coherence degree. In Eq. (30), the two slits wave functions $\psi_{\text {out } 1}$ and $\psi_{\text {out } 2}$ are calculated by the quantum approach (in Eqs. (24)(25)). In Refs. [7], the two slits wave functions are two Gaussian wave packets. The fringe visibility of $\nu$ is defined as $[7]$ :

$$
\nu=\frac{I_{\max }-I_{\min }}{I_{\max }+I_{\min }},
$$

$I_{\max }$ and $I_{\min }$ being the intensities corresponding to the central maximum and the first minimum next to it, respectively. The value for the fringe visibility of $\nu=0.59$ is obtained in Zeilinger et. al. experiment [20] $\left(I_{\max }=4076, I_{\min }=1050\right)$, and the quantum coherence degree $\Lambda_{t} \approx \nu[7]$.

\section{Numerical result}

Next, we present our numerical calculation of relative diffraction intensity. The main input parameters are: neutron mass $M=1.67 \times 10^{-27} \mathrm{~kg}$, the distance between slit and display screen $l=5 \mathrm{~m}$, the diffraction angle on $y z$ surface $\alpha=0 \mathrm{rad}$, the slit thickness $c=3.0 \times 10^{-5} \mathrm{~m}$, the neutron energy $E=3.3 \times 10^{-23} \mathrm{~J}$ (corresponding to neutron wave length $\lambda=20 \AA$ ) and Planck's constant $\hbar=1.055 \times 10^{-34}$ Js. The equations (24)-(30) are series for the integer $m$ and $n$. We find the series is convergence when $m \geq 600$ and $n \geq 10$, so we can make numerical calculation for equations (24)-(30). For single-slit experiment, the neutron wavelength $\lambda=20 \AA$, the slit width $a_{1}=90 \mu \mathrm{m}$. In our calculation, we take the same experiment parameters above, and the theoretical input amplitude parameter $A=2.45 \times 10^{4}$. From Eq. (27), we can obtain the diffraction intensity pattern, and it is shown in FIG. 3. In FIG. 3, the solid curve is our calculation result, and the dot curve is the experiment data [20]. From FIG. 3, we can find the calculation result is agreement with experiment data. For the double-slit diffraction, we consider two cases: coherence superposition and decoherence effect. For the coherence superposition, we can calculate the diffraction intensity from Eq. (28), and it is shown in FIG. 4. The experiment parameters are: the neutron wavelength $\lambda=20 \AA$, the first and second slit width $a_{1}=21.9 \mu m, a_{2}=22.5 \mu m$, the distance between the two slit $d=100 \mu m$. In our calculation, we take the same experiment parameters above, and the theoretical input parameters are superposition coefficients $c_{1}=0.397, c_{2}=0.918\left(\left|c_{1}\right|^{2}+\left|c_{2}\right|^{2}=1\right)$ and amplitude parameter $A=6.8 \times 10^{-2}$. In FIG. 4, the solid curve is our theoretical calculation, and the dot curve is the experiment data [20]. From the FIG. 4, we find that the theoretical result is in accordance with the experiment data, when the position $s$ is in the range of $|s| \geq 300 \mu m$. When the position $s$ is in the range of $|s| \leq 300 \mu m$, the theoretical result has a large discrepancy with the experiment data. We find the discrepancy can be eliminated when the decoherence effect is considered. From Eq. (30), we can obtain the diffraction intensity pattern and it is shown in FIG. 5. In calculation, superposition coefficients $c_{1}=0.397, c_{2}=0.918$, amplitude $A=6.8 \times 10^{-2}$ and quantum coherence degree $\Lambda_{t}=0.59$. In FIG. 5, the solid curve is our theoretical calculation, and the dot curve is the experiment data [20]. From FIG. 5, we can find when the decoherence effect is considered, the calculation result is in accordance with the experiment data, and the discrepancy between the theoretical result and experiment data can be eliminated.

\section{Conclusion}

In conclusion, we study neutron single and double-slit diffraction with quantum theory approach. The calculation result of single-slit diffraction is in accordance with the experiment data. For the double-slit diffraction, we study the diffraction intensity by two approaches, which are the coherence superposition and decoherence mechanism. When we consider the coherence superposition, the theoretical result has a large discrepancy with the experiment data. When we consider the decoherence mechanism, the theoretical result is in accordance with the experiment data, and the discrepancy between the theoretical result and 
experiment data has be eliminated. Otherwise, we think the approach has universal applicability, such as, it can also study electron, atom and molecular diffraction, and it can also be studied multi-slit and grating diffraction. 
1 O. Carnal and J. Mlynek, Phys. Rev. Lett. 662689 (1991)

${ }^{2}$ W. Schöllkopf and P. J. Toennies, Science 2661345 (1994)

3 M. Arudt, O. Nairz, J. Voss-Andreae, C. Kwller, G. Vander Zouw and A. Zeilinger, Nature 401680 (1999)

${ }^{4}$ O. Nairz, M. Arudt and A. Zeilinger, J. Mod. Opt. 472811 (2000)

${ }^{5}$ Kunze S, Dieckmann k and Rempe G, Phys. Rev. Lett. 782038 (1997)

${ }^{6}$ B. Brezger, L. Hackermuller, S. Uttenthaler, J. Petschinka, M. Arndt and A. Zeilinger, Phys. Rev. Lett. 88, 100404 (2002)

7 S. A. Sanz, F. Borondo and J. M. Bastiaans, Phys. Rev. A 71, 042103 (2005)

8 O. Carnal and J. Mlynek, Phys. Rev. Lett. 66, 2689 (1991)

9 W. Schöllkopf and P. J. Toennies, Science 266, 1345 (1994)

10 M. Arudt, O. Nairz, J. Voss-Andreae, C. Kwller, G.Vander Zouw and A. Zeilinger, Nature 401, 680 (1999)

11 O. Nairz, M. Arudt and A. Zeilinger, J. Mod. Opt. 47, 2811 (2000)

12 S. Kunze, K. Dieckmann and G. Rempe, Phys. Rev. Lett. 78, 2038 (1997)

13 X. Y. Wu, B. J. Zhang, H. B. Li, J. B. Lu, X. J. Liu, L. Wang, C. L. Zhang, B. Liu, X. H. Fan and Y. Q. Guo, Chin. Phys. Lett. 24, 2741 (2007)

14 Xiang-Yao Wu, Bai-Jun Zhang and Xiao-Jing Liu Chun-Li Zhang, Bing Liu and Yi-Qing Guo, FIZIKA B (Zagreb) 18, 4 (2009).

${ }^{15}$ Li Wang, Bai-Jun Zhang, Zhong Hua, Ji Li, Xiao-Jing Liu and Xiang-Yao Wu, Progress of Theoretical Physics 121, 685 (2009).

16 A. Viale, M. Vicari and N. Zanghi, Phys. Rev. A 68, 063610 (2003).

17 R. Tumulka, A. Viale and N. Zanghi, Phys. Rev. A 75, 055602 (2007)

18 C. Kiefer and E. Joos, Decoherence: Concepts and Examples, in Quantum Future, eds. P. Blanchard and A. Jadczyk (Springer, Berlin, 1998).

19 E. Joos and H.D. Zeh, Zeit. Phys. 59B, 223 (1985).

20 Anton Zeilinger, Roland Gahler, C. G. Shull and Walter Mampe, Rev. Mod. Phys. 60, 1067 (1988)

21 J.D. Jackson 1999 Classical Electrodynamics (Chichester: John Wiley-Sons) chap 10 p 579 


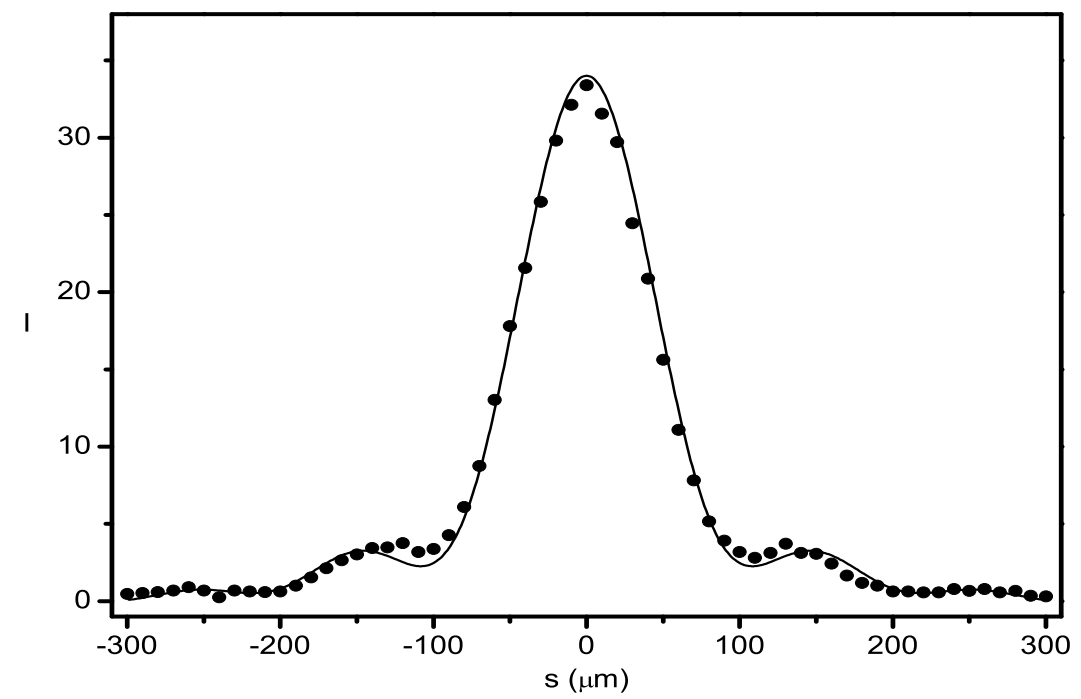

FIG. 3: Comparison between theoretical prediction from Eq. (27) (solid line) and experimental data taken from [20] (circle point) for neutron single-slit diffraction.

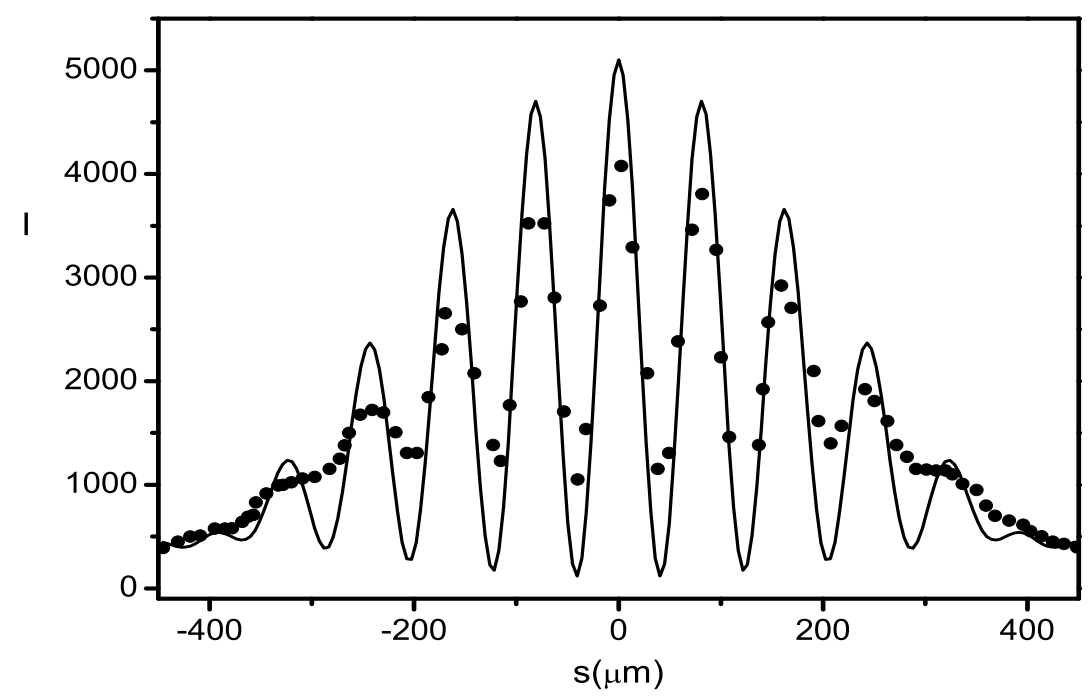

FIG. 4: Comparison between theoretical prediction from Eq. (28) (solid line) and experimental data taken from $[20]$ (circle point) for neutron double-slit diffraction, no including the decoherence effects. 


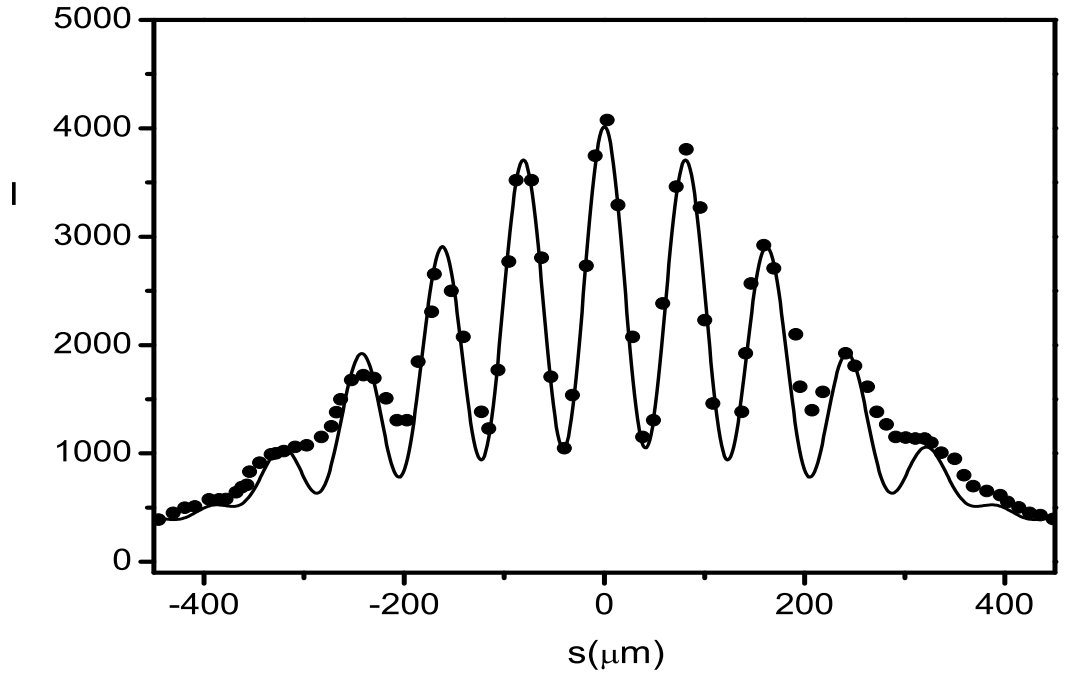

FIG. 5: Comparison between theoretical prediction from Eq. (28) (solid line) and experimental data taken from $[20]$ (circle point) for neutron double-slit diffraction, no including the decoherence effects. 